\title{
Miniaturization and Characterization of Metamaterial Resonant Particles
}

\author{
${ }^{1}$ Francisco Aznar, ${ }^{1}$ Jordi Bonache, ${ }^{1}$ Ferran Martín, ${ }^{2}$ Ekmel Ozbay, ${ }^{2} \mathrm{~K}$. Boratay Alici, ${ }^{3}$ Filiberto Bilotti, \\ ${ }^{3}$ Simone Tricarico, ${ }^{3}$ Lucio Vegni, ${ }^{4}$ Juan D. Baena, ${ }^{4}$ Lukas Jelinek and ${ }^{4}$ Ricardo Marqués \\ ${ }^{I}$ GEMMA/CIMITEC, Departament d'Enginyeria Electrònica, Universitat Autònoma de Barcelona, 08193 BELLATERRA, Spain \\ ferran.martin@uab.es \\ ${ }^{2}$ Nanotechnology Research Center, Bilkent University, Ankara 06800, Turkey \\ ${ }^{3}$ Department of Applied Electronics, University "Roma Tre", 00146 Rome, Italy \\ ${ }^{4}$ Departamento de Electrónica y Electromagnetismo, Universidad de Sevilla, 41012 Sevilla, Spain
}

\begin{abstract}
This paper is focussed on the miniaturization and characterization of semi-lumped resonators, of interest for the synthesis of metamaterial-based structures such as metamaterial transmission lines, frequency selective surfaces, absorbers, and radiating elements, among others. The particles consist on metallic patterns etched on a dielectric and are inspired on the split ring resonator, SRR (that is, the formerly resonant particle used for the synthesis of left handed metamaterials). The different strategies for miniaturization are discussed and examples are given. It is shown that by using two metallic levels connected through vias it is possible to achieve very small electrical size for the particles (namely, below $\lambda / 100$, where $\lambda$ is the wavelength in the considered substrate at resonance). A method to determine the electrical parameters of the resonators in metamaterial transmission line configurations is also presented, and the possibility to determine the characteristics of the isolated particles is discussed. Finally, examples of application of this technique are presented. This work is of interest for the synthesis of effective media metamaterials based on resonant elements.
\end{abstract}

\section{INTRODUCTION}

Effective media metamaterials are artificial structures based on periodic inclusions of metals and/or dielectrics that exhibit controllable characteristics. Rather than being based on the composition of the elements forming the medium, the electromagnetic (or optical) properties of effective media metamaterials are based on their structure. Thanks to this controllability, it is possible to synthesize media with unusual properties, including negative refractive index media [1], or invisible cloaks [2], among others. Probably, the most relevant works that have led to the explosion of metamaterials are due to Pendry and co-workers [3], Smith and co-workers [4], and Veselago [5]. In [3], the split ring resonator (SRR) - see Fig. 1a was presented as a small size resonant particle useful for the synthesis of artificial (anisotropic) media with negative effective permeability. In [4], it was synthesized the first left handed medium by combining SRRs with metallic posts (the latter providing the required negative permittivity). Although highly anisotropic (the structure presented in [4] is a bulk onedimensional left-handed medium), it represented the first synthesis of a medium with simultaneous negative permittivity and permeability (the properties of those media were already anticipated by Veselago in 1968 [5], and have been experimentally demonstrated from the beginning of this millennium - see [6-9] and references therein).

Multiple works have been devoted to the synthesis of functional artificial structures based on SRRs. This includes, metamaterial transmission lines (and their microwave applications [10,11]), admittance surfaces [12], absorbers [13], radiating elements [14,15], imaging devices [16], etc. (see [9] for more details). One key aspect in many of these applications is the miniaturization of the resonant particles. To reduce the electrical size of SRRs, it is necessary to enhance the coupling between the individual rings [17]. In a uni-planar configuration (edge coupling), the limit is given by the minimum distance between etched metals, which is of the order of 50-100 $\mu \mathrm{m}$ for typical microwave substrates and etching systems (drilling machine or photo/etching system). With such critical dimensions, it is not possible to achieve particle diameters smaller than $\lambda / 10$. The electrical size of the particle can be reduced by etching the individual rings face-toface at different metal levels (broadside coupling), see Fig. 1(c). By using thin dielectrics, the distributed capacitance between the rings can be increased and the first resonance frequency significantly reduced (SRR diameters of about $\lambda / 30$ have been demonstrated by using this approach [18]). Another possibility is to modify the topology so that the slits are etched in the same position and the inner and outer rings are cross connected $[19,20]$. The resulting topology is indeed a two-turn spiral (Fig. 1b) whose resonance frequency is half the resonance frequency of the SRR (the particle inductance is preserved but the capacitance is enhanced by a factor of four [19]).

In this paper, different strategies for particle miniaturization are presented. Miniaturization levels superior to $\lambda / 100$ are demonstrated. In some cases, due to their complexity, it is difficult to derive analytical models for such particles. For this reason, we have developed a method to obtain the electrical parameters of the resonators when they are coupled to transmission lines. The possibility to obtain the parameters of the decoupled particles is discussed, and examples of particle characterization are provided. 


\section{STRATEGIES FOR PARTICLE MINIATURIZATION}

The strategies for the miniaturization of metamaterial resonators either consist on using two metallic levels connected by vias and/or increasing the number of elements constituting the structure. For instance, it is possible to significantly reduce the electrical size of the resonators by implementing a two-turn spiral resonator with either turn etched face-to-face at both sides of a thin substrate and connected through a via (Fig 1f). By means of this topology, we take benefit of the broadside coupling as well as the higher capacitance inherent to the topology, as has been discussed in the previous section with regard to the two turn uni-planar spiral resonator (Fig 1b). We can further reduce the particle size by increasing the number of turns, as is illustrated in Fig. 1(g). With this particle we achieve a high level of miniaturization, which is of the order of $\lambda / 100$ provided a substrate with $0.127 \mathrm{~mm}$ thickness is considered.

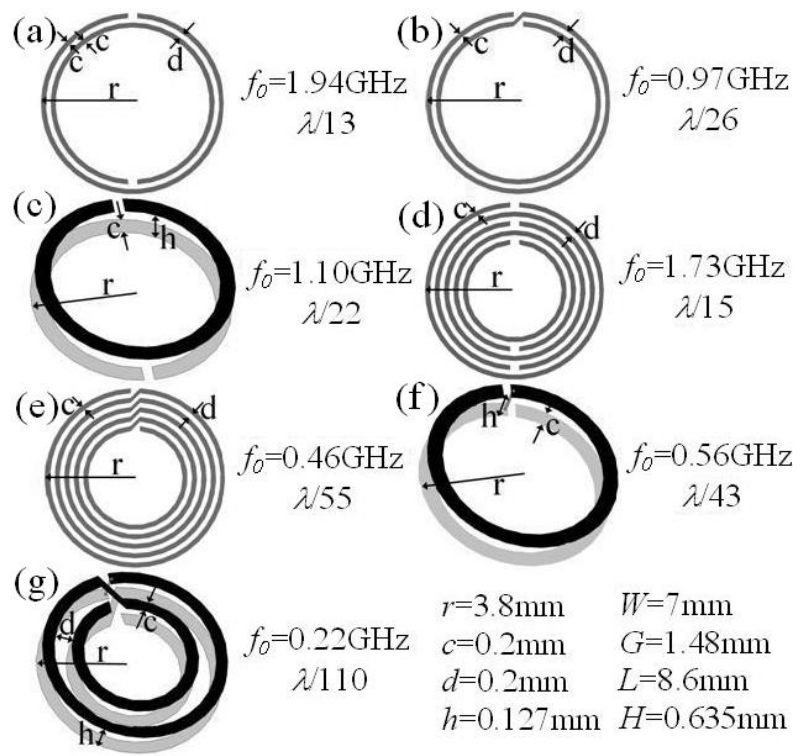

Fig. 1 Topologies of the different resonators considered, and indication of the resonance frequency and size obtained as is explained in the text with the structure depicted in Fig. 2. The considered substrate is Rogers RO3010 with dielectric constant $\varepsilon_{r}=10.2$. Relevant dimensions are: metal strips width $c=0.2 \mathrm{~mm}$, distance between strips $d=0.2 \mathrm{~mm}$, external radius $r=3.8 \mathrm{~mm}$, substrate thickness $h=0.127 \mathrm{~mm}$ (for resonators formed by two metal layers). For the CPW structure the central strip width is $W=7 \mathrm{~mm}$, the width of the slots is $G=1.35 \mathrm{~mm}$, the length of the line is $L=8.6 \mathrm{~mm}$ and the substrate thickness $H=0.635 \mathrm{~mm}$.

By increasing the number of turns of the spiral resonator in a uni-planar metal configuration (Fig 1e), the resonance frequency can be significantly reduced, although it tends to saturate. It is also possible to introduce further rings to the topology of the SRR, as Fig. 1(d) illustrates. This also reduces the electrical size due to multiple coupling. The different topologies discussed, as well as their electrical size, are all depicted in Fig. 1. To obtain the electrical size of these particles we have coupled them to a CPW transmission line, as is depicted in Fig. 2, and we have obtained the simulated transmission coefficient of the structure (by using the Agilent Momentum commercial software). The transmission zero coincides with the resonance frequency of the particle. Thus, we can easily obtain the particle size in terms of the wavelength at resonance. We must mention that the resonance frequency obtained by following this procedure corresponds to the resonance of the particle coupled to the line. That is, the presence of the CPW affects the particle to some extent (this aspect will be discussed later). Nevertheless, in many applications, the particles are coupled to a line and it is of interest to infer the parameter under these circumstances.

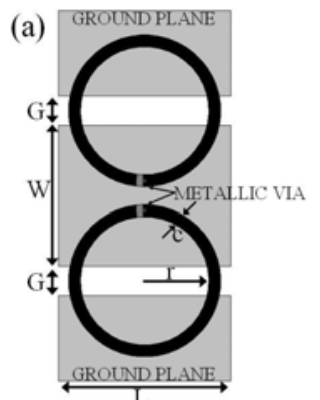

(b)

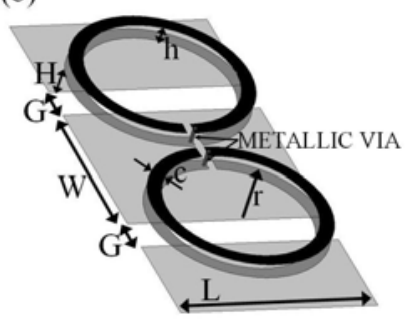

Fig. 2 Typical configuration of a CPW loaded with a pair of metamaterial resonators (Fig 1f) used to determine their resonance frequency, top (a) and 3D (b) view. Relevant dimensions are indicated in Fig. 1.

\section{PARAMETER EXTRACTION}

The structure of Fig. 2 can be described by the circuit model of Fig. 3(a) [21]. The resonators are described by the resonant tank $L_{s}-C_{s}, M$ is the mutual inductive coupling between the line and the resonators, $L$ is the line inductance and $C$ the line capacitance. Notice that, contrary to the model reported in [21], in the model of Fig. 3(a) we have not used the magnetic wall concept since it is not actually necessary. This circuit model can be transformed to that of Fig. 3(b) with the following transformations:

$$
\begin{gathered}
C_{s}{ }^{\prime}=\frac{L_{s}}{2 \omega_{o}{ }^{2} M^{2}} \\
L_{s}{ }^{\prime}=2 \omega_{o}{ }^{2} M^{2} C_{s} \\
L^{\prime}=L-L_{s}{ }^{\prime}
\end{gathered}
$$

where $\omega_{o}=2 \pi f_{o}, f_{o}$ being the resonance frequency of the resonators:

$$
f_{o}=\frac{1}{2 \pi} \sqrt{\frac{1}{L_{s}^{\prime} C_{s}^{\prime}}}=\frac{1}{2 \pi} \sqrt{\frac{1}{L_{s} C_{s}}}
$$

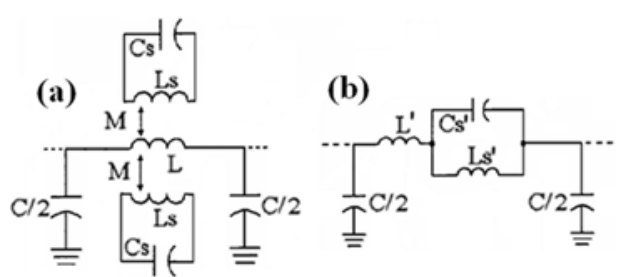

Fig. 3 Lumped element equivalent circuit model of a transmission line loaded with a pair of metamaterial resonant particles (a) and transformed model obtained by the procedure indicated in the text (b). 
To determine the parameters of the circuit model of Fig. 3(b) we need four conditions. From the representation of the reflection coefficient, $S_{11}$, in the Smith Chart, two conditions are obtained. On one hand, we can determine the frequency that nulls the series reactance, $f_{z}$, from the intercept of $S_{11}$ with the unit conductance circle. This is obvious since at this frequency, the real part of the admittance seen from the ports is simply the admittance of the opposite port, that is, $Y_{o}=1 / Z_{o}=(50 \Omega)^{-1}=0.02 \mathrm{~S}$. This frequency is given by the following expression:

$$
f_{z}=\frac{1}{2 \pi} \sqrt{\frac{1}{L_{s}^{\prime} C_{s}^{\prime}}+\frac{1}{L^{\prime} C_{s}^{\prime}}}
$$

On the other hand, the susceptance of the unit cell seen from the ports at $f_{z}$, which can be inferred from the Smith Chart, is:

$$
B\left(\omega_{z}\right)=\frac{C \omega_{z}}{2}
$$

with $\omega_{z}=2 \pi f_{z}$. Another condition is obtained from the representation of transmission coefficient, $S_{2 l}$, since the transmission zero frequency, which coincides with the resonance frequency of the particles and can be easily identified, is given by expression (4). Finally, another condition can be deduced from the phase of the transmission coefficient, $\phi_{S 21}$. At the frequency where $\phi_{S 21}=90^{\circ}, f_{\pi / 2}$, the electrical length of the unit cell, $\phi=\beta l$ ( $\beta$ being the phase constant and $l$ the length of the unit cell), is $\phi\left(f_{\pi / 2}\right)=-90^{\circ}$. Since the dispersion relation of a periodic structure consisting of cascaded unit cells as those of Fig. 2 is given by:

$$
\cos \phi=1+\frac{Z_{s}(\omega)}{Z_{p}(\omega)}
$$

it follows that

$$
Z_{s}\left(\omega_{\pi / 2}\right)=-Z_{p}\left(\omega_{\pi / 2}\right)
$$

with $\omega_{\pi / 2}=2 \pi f_{\pi / 2}$. Expressions (4)-(6) and (8) are the four conditions needed to univocally determine the circuit parameters of Fig. 3(b). To illustrate the accuracy of the reported method and the validity of the model, we have applied it to determine the parameters of the structure depicted in Fig. 2. Fig. 4 shows the comparison between the electromagnetic and circuit simulation (the circuit parameters have been inferred from the simulated S-parameters of the structure, but it is also possible to infer them from measurement). Good agreement has been obtained, indicating the validity of the circuit model and parameter extraction.

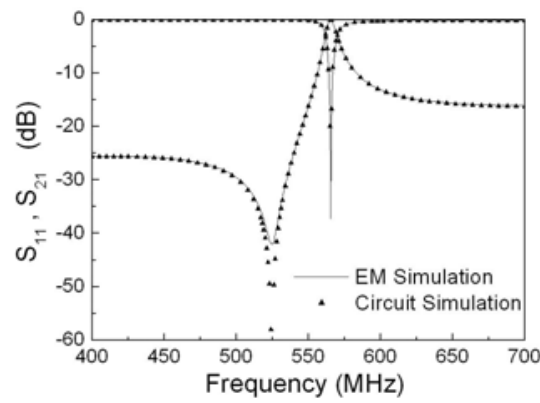

Fig. 4 Frequency response of the structure of Fig. 2 obtained by electromagnetic and circuit simulation. The dimensions are depicted in Fig.1 and the circuit parameters in Table I(f).

\section{APPLICATION OF THE PARAMETER EXTRACTON TECHNIQUE TO THE MINIATURIZED RESONATORS}

The method reported in the previous section has been applied to obtain the parameters of the circuit model of Fig. 3(b), corresponding to the CPW transmission lines loaded with the resonant elements considered in Section II. The results are given in Table I. Additionally, in this table are given the values of the elements of the original circuit (Fig. 3a), which have been inferred from transformation (1)-(3) by considering $M=L \cdot f$, where $f$ is the fraction of the slots occupied by the resonant elements. This assumption is roughly valid if (i) the resonant elements can be described through a quasistatic analysis, and (ii) the magnetic flux lines generated by the line in the region of the resonators penetrate the whole resonator area. To satisfy the latter requirement it is necessary to use moderate or narrow substrates.

TABLE I

ELEMENT VALUES FOR THE DIFFERENT RESONATORS CONSIDERED IN FIG. 1

\begin{tabular}{|l|c|c|c|c|c|c|c|}
\cline { 2 - 8 } \multicolumn{1}{c|}{} & (a) & (b) & (c) & (d) & (e) & (f) & (g) \\
\hline $\mathrm{C}(\mathrm{pF})$ & 2.51 & 2.19 & 2.11 & 3.55 & 3.00 & 2.05 & 2.45 \\
\hline \multicolumn{8}{|c|}{ For the circuit model in Fig. 3(b) } \\
\hline $\mathrm{L}^{\prime}(\mathrm{nH})$ & 1.93 & 2.13 & 2.00 & 1.76 & 2.18 & 2.21 & 2.40 \\
\hline $\mathrm{L}_{\mathrm{S}}{ }^{\prime}(\mathrm{nH})$ & 0.37 & 0.42 & 0.37 & 0.44 & 0.52 & 0.38 & 0.53 \\
\hline $\mathrm{C}_{\mathrm{S}}{ }^{\prime}(\mathrm{pF})$ & 17.9 & 63.4 & 56.4 & 19.4 & 231 & 206 & 979 \\
\hline \multicolumn{8}{|c|}{ For the circuit model in Fig. 3(a) } \\
\hline $\mathrm{M}(\mathrm{nH})$ & 1.72 & 1.90 & 1.98 & 1.02 & 1.26 & 2.18 & 2.17 \\
\hline $\mathrm{L}(\mathrm{nH})$ & 2.31 & 2.55 & 2.37 & 2.20 & 2.71 & 2.60 & 2.92 \\
\hline $\mathrm{L}_{\mathrm{S}}(\mathrm{nH})$ & 15.7 & 17.2 & 21.2 & 4.81 & 6.04 & 24.7 & 17.9 \\
\hline $\mathrm{C}_{\mathrm{S}}(\mathrm{pF})$ & 0.43 & 1.55 & 0.99 & 1.76 & 20.0 & 3.22 & 28.9 \\
\hline
\end{tabular}

It is worth mentioning that the estimated resonator parameters correspond to the particle in the presence of the $\mathrm{CPW}$ on the opposite substrate side. However, it is possible to estimate the parameters of the isolated particle (that is, without the presence of the CPW metal level). To this end we make the following assumption: the resonators are considerably distant to the CPW. Under this conditions, the capacitance $C_{s}$ roughly coincides with the capacitance of the isolated particle, $C_{I}$. The inductance of the isolated particle can be inferred from the method of images according to:

$$
L_{I}=L_{s}+M^{\prime}
$$

where $M^{\prime}$ is the mutual coupling between the resonator and its image.

We have applied this procedure to the SRR particle since the analytical model of this particle is well known [18]. The parameters of the isolated resonator coincide to a good approximation with those inferred from the method reported in [18] for substrate thicknesses in the vicinity of $0.6 \mathrm{~mm}$ $0.8 \mathrm{~mm}$. For thicker substrates, the values inferred from the analytical model can also be obtained by varying (decreasing) the filling factor $f$ (that is, the second assumption for the validity of $M=L \cdot f$ is not satisfied). For thinner substrates the distributed capacitance between the individual rings of the SRR is influenced by the presence of the CPW metal level, and this complicates $C_{I}$ calculation. In deriving (9), it has been considered that the effect of the slots $(\mathrm{CPW})$ is negligible. 
According to these words, to accurately derive the parameters of the isolated particles, we need to know $M$ accurately, and this is not so simple because we can not consider that the optimized substrate thicknesses for the SRR hold for other particles. Indeed, this has been corroborated since it has been found that the optimized substrate thickness for the broadside coupled SRR is different. Nevertheless, for many applications based on the transmission line model of Fig. 3(b), the reported parameter extraction techniques is very valuable, since we are able to determine not only the resonator parameters, but also the line parameters very accurately. For the isolated particles, the method is only able to provide approximate values of the resonator parameters. This method is of special interest for those structures based on complex resonators which can not be easily modelled.

\section{CONCLUSIONS}

In conclusion, we have proposed small size planar resonators of interest for the synthesis of effective media metamaterials. Dimensions as small as $\lambda / 100$ ( $\lambda$ being the wavelength at resonance) have been demonstrated. We have also proposed a technique for the determination of resonator parameters when they are coupled to a planar transmission line. This is of interest in many applications based on transmission line metamaterials. The technique has been applied to various resonant particles. The procedure for the determination of the parameters of the isolated particles has also been discussed, and its limitations have been pointed out. This paper is of interest for the microwave community involved in metamaterial synthesis and their applications.

\section{ACKNOWLEDGMENT}

This work has been supported by MEC, Spain, through project contract TEC2007-68013-C02 METAINNOVA, and by the European Commission (VI Framework Program) contract NoE. 500252-2 METAMORPHOSE. Special thanks are also given to CIDEM (Generalitat de Catalunya, Spain) for funding CIMITEC.

\section{REFERENCES}

[1] R.A. Shelby, D.R. Smith and S. Schultz, "Experimental verification of a negative index of refraction", Science, vol. 292, pp. 77-79 (2001).

[2] D. Schurig, J. J. Mock, B. J. Justice, S. A. Cummer, J. B. Pendry, A. F. Starr, D. R. Smith1, "Metamaterial electromagnetic cloak at microwave frequencies", Science, vol. 314, pp. 977-980, Nov. 2006.

[3] J.B. Pendry, A.J. Holden, D.J. Robbins and W.J. Stewart, "Magnetism from conductors and enhanced nonlinear phenomena", IEEE Transactions Microwave Theory Tech., vol. 47, pp. 2075-2084 (1999).

[4] D.R. Smith, W.J. Padilla, D.C. Vier, S.C. Nemat-Nasser and S. Schultz, "Composite medium with simultaneously negative permeability and permittivity", Phys. Rev. Lett., vol. 84, pp. 4184-4187 (2000).
[5] V.G. Veselago, "The electrodynamics of substances with simultaneously negative values of $\varepsilon$ and $\mu$ ", Sov. Phys. Usp. vol. 10, pp. 509-514 (1968)

[6] G.V. Eleftheriades and K.G. Balmain, Negative refraction metamaterials: fundamental principles and applications, John Wiley \& Sons Inc., New Jersey 2005.

[7] N. Engheta and R. W. Ziolkowski Edts., Metamaterials. Physics and Engineering Explorations, John Wiley \& Sons Inc., New Jersey 2006.

[8] C. Caloz and T. Itoh, Electromagnetic Metamaterials: Transmission Line Theory and Microwave Applications, John Wiley \& Sons Inc., New Jersey, 2006.

[9] R. Marqués, F. Martin and M. Sorolla, Metamaterials with Negative Parameters: Theory, Design and Microwave Applications, John Wiley \& Sons Inc., New Jersey, 2008.

[10] F. Martín, F. Falcone, J. Bonache, T. Lopetegi, R. Marqués and M. Sorolla, "Miniaturized CPW stop band filters based on multiple tuned split ring resonators", IEEE Microwave and Wireless Components Letters, vol. 13, pp. 511-513, December 2003.

[11] F. Falcone, F. Martín, J. Bonache, R. Marqués, T. Lopetegi and M. Sorolla, "Left handed coplanar waveguide band pass filters based on bi-layer split ring resonators", IEEE Microwave and Wireless Components Letters, vol. 14, pp. 10-12, January 2004.

[12] R. Marqués, J.D. Baena, M. Beruete, F. Falcone, T. Lopetegi, M. Sorolla, F. Martín and J. García-García, "Ab initio analysis of frequency selective surfaces based on conventional and complementary split rings resonators", Journal of Optics A, vol. 7, pp. S38-S43, 2005.

[13] F. Bilotti, L. Nucci, and L. Vegni, "An SRR Based Microwave Absorber", Microw. Opt. Techn. Let., vol. 48, No. 11, pp. 2171-2175, Nov. 2006

[14] F. Bilotti, A. Alù, and L. Vegni, "Design of Miniaturized Metamaterial Patch Antennas with $\mu$-Negative Loading," IEEE T. Antenn. Propag., vol. AP-56, 2008 (to appear)

[15] I. Arnedo, J. Illescas, M. Flores, T. Lopetegi, M.A.G. Laso, F. Falcone, J. Bonache, J. García-García, F. Martín, J.A. Marcotegui, R. Marqués and M. Sorolla, "Forward and Backward Leaky Wave Radiation in Split Ring Resonator based Metamaterials", IET Microwaves Antennas and Propagation, vol. 1 (1), pp. 65-68, February 2007.

[16] M.J. Freire and R. Marqués, "Near-field imaging in the megahertz range by strongly coupled magnetoinductive surfaces: experiment and ab initio analysis", J. Appl. Phys., vol. 100, pp. 063105, 2006.

[17] J. García-García, F. Martín, J.D. Baena, R.Maques, "On the resonances and polarizabilities of split rings resonators", J. Appl. Phys., vol. 98, pp. 033103-1-9, September 2005.

[18] R. Marqués, F. Mesa, J. Martel and F. Medina, "Comparative analysis of edge and broadside couple split ring resonators for metamaterial design. Theory and Experiment", IEEE T. Antenn. Propag., vol. 51, pp. 2572-2581, (2003).

[19] J.D. Baena, R. Marqués, F. Medina, J. Martel "Artificial magnetic metamaterial design by using spiral resonators", Phys. Rev. B, vol. 69 , pp. 014402-1-5, Jan. 2004.

[20] F. Falcone, F. Martín, J. Bonache, M.A.G. Laso, J. García-García, J.D. Baena, R. Marqués, M. Sorolla, "Stop band and band pass characteristics in coplanar waveguides coupled to spiral resonators", Microw. Opt. Techn. Let., vol. 42, pp. 386-388, September 2004.

[21] F. Martín, F. Falcone, J. Bonache, R. Marqués and M. Sorolla, "Split ring resonator based left handed coplanar waveguide", Appl. Phys. Lett., vol. 83 , pp. 4652-4654, December 2003. 